\title{
Hybrid reconstruction of the aortic arch: A 15-year follow-up
}

\author{
Charles A. Dietl, MD, ${ }^{a}$ Mark Langsfeld, MD, ${ }^{b}$ Adriana M. Sánchez, MD,${ }^{c}$ and Von G. Samedi, MD, PhD, \\ Albuquerque, NM
}

\footnotetext{
From the Divisions of ${ }^{\mathrm{a}}$ Cardiothoracic Surgery and ${ }^{\mathrm{b}}$ Vascular Surgery, Department of Surgery, and ${ }^{\mathrm{c}}$ Department of Pathology, University of New Mexico Health Sciences Center, Albuquerque, NM.

Disclosures: Authors have nothing to disclose with regard to commercial support.

Received for publication April 5, 2017; revisions received May 25, 2017; accepted for publication July 31, 2017; available ahead of print Sept 12, 2017.

Address for reprints: Charles A. Dietl, MD, Division of Cardiothoracic Surgery, Department of Surgery, University of New Mexico, 2-ACC, MSC 10 5610, Albuquerque, NM 87131-0001 (E-mail: CDietl@salud.unm.edu; cdietl@comcast.net).

J Thorac Cardiovasc Surg 2017;154:e95-7

$0022-5223 / \$ 36.00$

Copyright (c) 2017 by The American Association for Thoracic Surgery

http://dx.doi.org/10.1016/j.jtcvs.2017.07.079
}

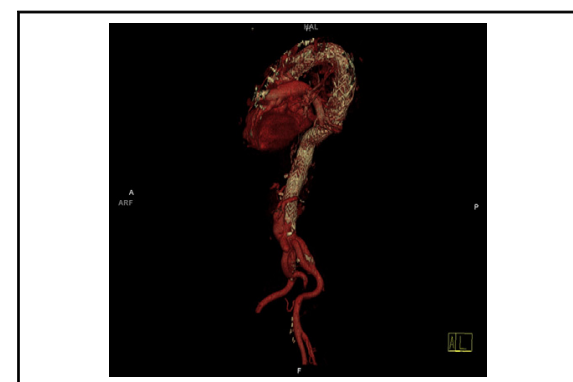

$3 \mathrm{D}$ reconstruction of the aorta 15 years after postoperative hybrid reconstruction of aortic arch.

\section{Central Message}

This study presents the case of a patient who underwent off-pump hybrid reconstruction of the aortic arch in 2001 and has been followed up for 15 years.

See Editorial Commentary page e99.
Conventional surgical therapy of aortic arch aneurysms is associated with significant morbidity and mortality, mainly from neurologic complications and the sequelae of deep hypothermic circulatory arrest. We present the case of a patient who underwent hybrid aortic arch repair with supra-aortic branch revascularization and subsequent endovascular stent-graft deployment and has been followed up for 15 years.

\section{CLINICAL SUMMARY}

A 44-year-old man with Marfan syndrome, who had previously undergone emergency replacement of the supracoronary ascending aorta for a type A acute aortic dissection in April 2001, presented with interscapular pain in December 2001. Computed tomographic angiography revealed a $6.5-\mathrm{cm}$ aneurysm of the distal aortic arch involving the origin of the left subclavian and left common carotid arteries. Conventional surgical resection with cardiopulmonary bypass and deep hypothermic circulatory arrest was offered, with off-pump aortic arch reconstruction and endovascular stent grafting offered as an alternative approach. The patient preferred the second option because of fear of neurologic complications. The procedure, which was performed off pump, was described in detail in our article published in 2003. ${ }^{1}$ A stent-graft device (Medtronic, Minneapolis, Minn) was used off label under physician Investigational Device Exemption study. The patient had an uneventful operation and postoperative recovery.

Follow-up computed tomographic angiography obtained at 12 months revealed that the aneurysmal sac was completely thrombosed, except for a small type $1 \mathrm{~b}$ distal endoleak, for which the patient underwent thoracic endovascular aortic repair. In October 2008, the patient was seen with a Crawford extent III thoracoabdominal aortic aneurysm with chronic dissection. He underwent hybrid reconstruction of the thoracoabdominal aortic aneurysm, consisting of open replacement of suprarenal aorta to

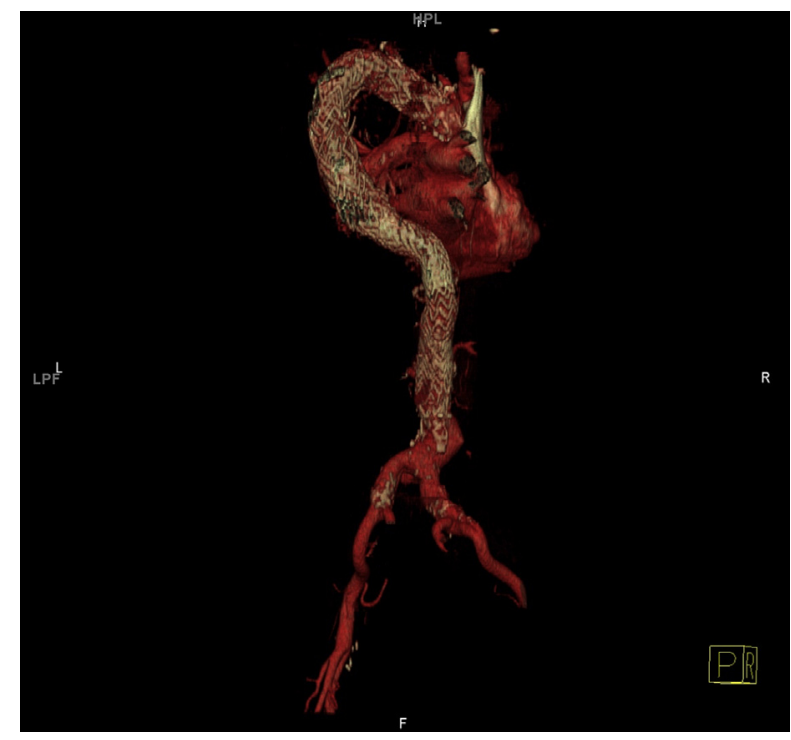

FIGURE 1. Computed tomographic angiography with 3-dimensional reconstruction of the aorta 15 years after hybrid reconstruction of aortic arch and 8 years after hybrid reconstruction of thoracoabdominal aorta. 


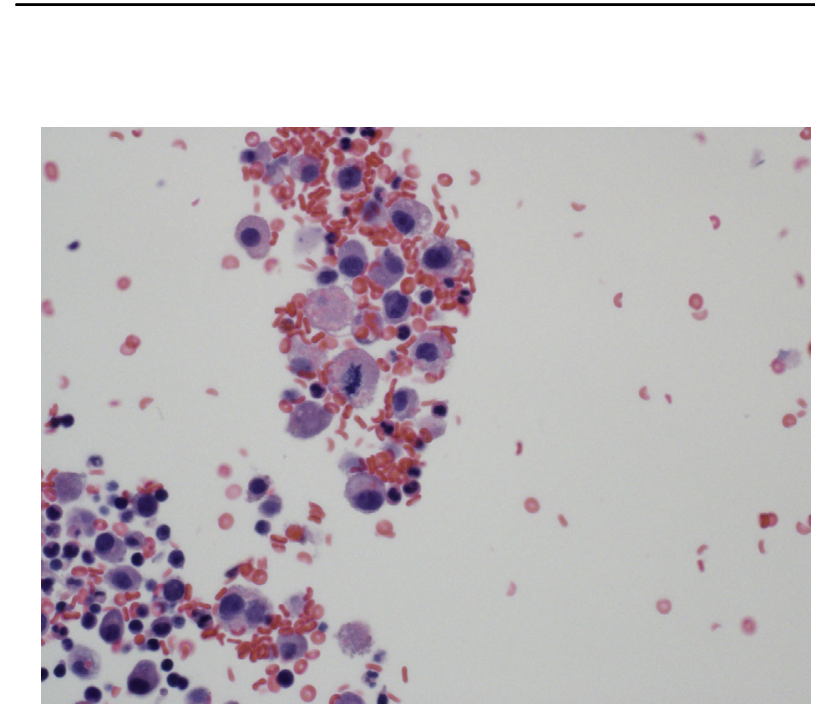

FIGURE 2. Malignant cells aspirated from the patient's left neck mass. Intracytoplasmic vacuoles and intranuclear inclusions are present (hematoxylin and eosin stains). A mitotically active cell is seen in the center. The diagnosis of epithelioid angiosarcoma was confirmed by positive immunohistochemical testing for vascular markers CD31 and ERG (not shown).

bifurcation with 28-mm Hemashield graft (MAQUET Cardiovascular, LLC, Wayne, NJ) and open debranching procedure to celiac, superior mesenteric artery and both renal arteries, combined with thoracic endovascular aortic repair of the thoracoabdominal aortic aneurysm.

In 2014 the patient had severe left-sided headaches; these were attributed to severe stenosis of the left common carotid artery graft and retrograde flow in the left vertebral artery, suspected to cause an endoleak responsible for an enlarging 9-cm aneurysm sac. He underwent a retropharyngeal right common carotid artery-to-left common carotid artery bypass with a ringed $8-\mathrm{mm}$ polytetrafluoroethylene graft, plus ligation of the left vertebral artery, and a left common carotid artery-to-left subclavian artery bypass with a ringed 8-mm polytetrafluoroethylene graft. Subsequently, the patient's headaches resolved.

The patient was reevaluated in December 2016 because he was reporting low back pain and a new mass had developed in his left neck. The mass was neither tender nor pulsatile on examination. Computed tomographic angiography of the neck, chest, abdomen, and pelvis revealed no pseudoaneurysms and no endoleaks (Figure 1). A conglomerate of enlarged left cervical, axillary, mediastinal, subcarinal, and retroperitoneal lymph nodes was seen, however, in addition to multiple bilateral lung nodules and lysis and loss of vertebral body height of T11 and T12, likely representing metastatic disease.

A needle biopsy of the left neck mass showed an incohesive population of large, highly atypical epithelioid cells with abundant cytoplasm and enlarged irregular nuclei with prominent nucleoli, consistent with epithelioid angiosarcoma (Figure 2). The patient's clinical course was complicated with progressive respiratory failure, and the

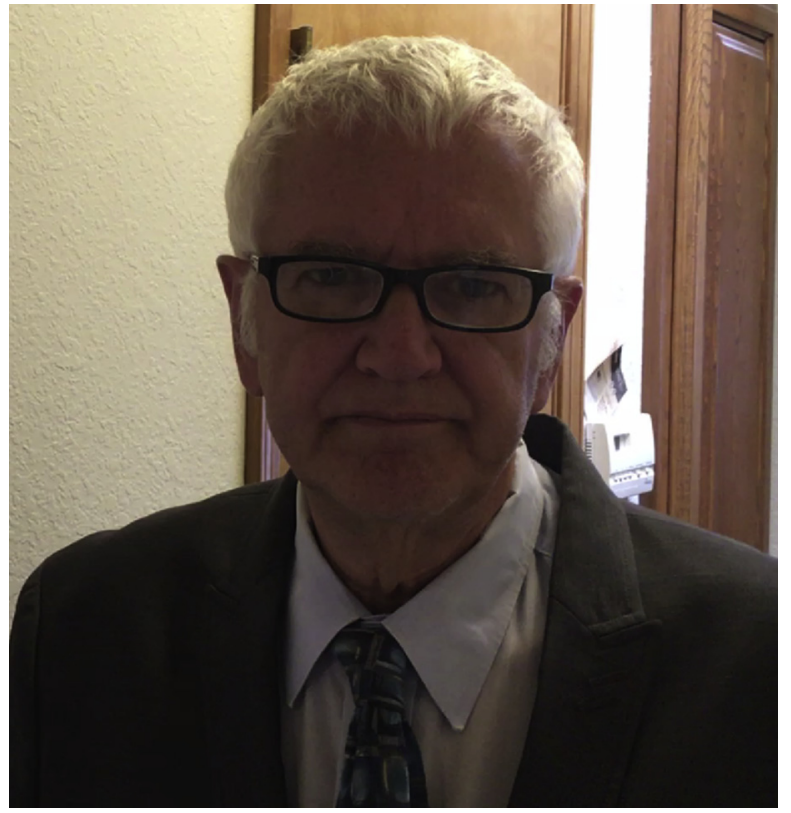

VIDEO 1. Description of surgical technique used for hybrid reconstruction of the aortic arch. Video available at: http://www.jtcvsonline.org/ article/S0022-5223(17)31775-0/fulltext.

patient died in December 2016, 15 years after he had undergone off-pump hybrid reconstruction of the aortic arch.

\section{DISCUSSION}

Conventional surgical therapy of aortic arch aneurysms consists of aortic arch replacement requiring cardiopulmonary bypass and deep hypothermic circulatory arrest. This method is associated with significant morbidity and mortality, mainly as a result of neurologic complications and the sequelae of deep hypothermic circulatory arrest.

The first 2 patients who underwent successful off-pump hybrid reconstruction of the aortic arch in 2001 were reported on by our group in 2003. ${ }^{1}$ To our knowledge, there are no previous reports in the literature of endovascular implantation of a stent graft to exclude the entire aortic arch combined with a debranching procedure without using cardiopulmonary bypass or deep hypothermic circulatory arrest (Video 1).

This approach is a reasonable treatment option for aortic arch aneurysms, especially in high-risk patients, and has been adopted by many centers for treatment of aortic arch aneurysms, specially in high-risk patients..$^{2-5}$ In summary, hybrid aortic arch repair with supra-aortic branch revascularization and subsequent endovascular stent-graft deployment has evolved as a less-invasive alternative treatment option with promising results.

\section{References}

1. Dietl CA, Kasirajan K, Pett SB, Wernly JA. Off-pump management of aortic arch aneurysm by using an endovascular thoracic stent graft. J Thorac Cardiovasc Surg. 2003; 126:1181-3. 
2. Szeto WY, Bavaria JE, Bowen FW, Woo EY, Fairman RM, Pochettino A. The hybrid total arch repair: brachiocephalic bypass and concomitant endovascular aortic arch stent graft placement. J Card Surg. 2007;22:97-102; discussion 103-4.

3. Weigang E, Parker J, Czerny M, Peivandi AA, Dorweiler B, Beyersdorf F, et al. Endovascular aortic arch repair after aortic arch de-branching. Ann Thorac Surg. 2009;87:603-7.
4. Bavaria J, Vallabhajosyula P, Moeller P, Szeto W, Desai N, Pochettino A. Hybric approaches in the treatment of aortic arch aneurysms: postoperative and midterm outcomes. J Thorac Cardiovasc Surg. 2013;145(3 Suppl):S85-90.

5. Moulakakis KG, Mylonas SN, Markatis F, Kotsis T, Kakisis J, Liapis CD. A systematic review and meta-analysis of hybrid aortic arch replacement. Ann Cardiothorac Surg. 2013;2:247-60. 